\title{
PERSPECTIVA DISCIPLINAR DE ENFERMERÍA EN LA OBRA DE SAN PEDRO CLAVER
}

Juan Guillermo Rojas', José Siles González

'Profesor Facultad de Enfermería,

U. de A., Medellín-Colombia.

${ }^{2}$ Catedrático Departamento de Enfermería, U. de Alicante.

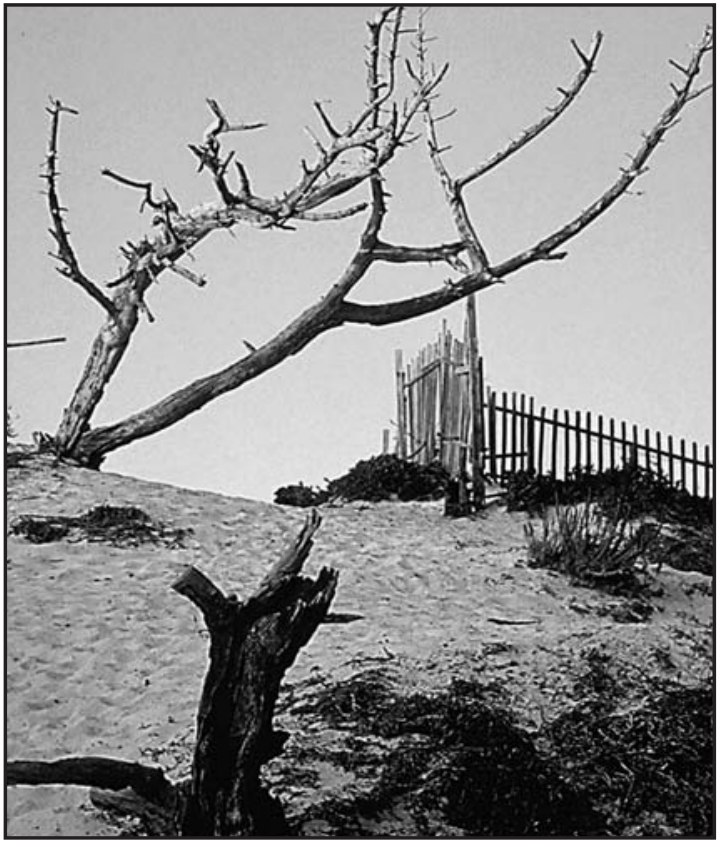

NURSING DISCIPLINE PERSPECTIVE ON ST. PETER CLAVER'S WORK

\section{SUMMARY}

$\mathrm{N}$ ursing historical development has been closely linked to human history, because care of life and health have been essential activities in necessities supply process. Nursing historical count, refers to facts, events and important figures that in different contexts have marked patterns for professional development.

Nowadays, nursing history teaching and learning became very important as a strategy to advance towards future with acknowledge of social and political background which have brought about nursing survival, development and advance as profession and discipline.

In this paper, a description about Father Pedro
Claver actions during his stay at Colonial Cartagena and an analysis are carried out, by means of epistemologic framework with the purpose to determine his philosophical perspective on care given to black slaves, and that have influenced throughout the time Colombian nursing development.

Key words: Nursing, Nursing Research, History of Nursing.

PERSPECTIVA DISCIPLINAR DA ENFERMAGEM NA OBRA DE SAO PEDRO CLAVER

\section{RESUMO}

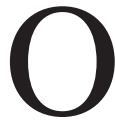
desenvolvimento histórico da enfermagen tem estado intimamente unido à história da humanidade, porquanto o cuidado da vida e a saúde, foram actividades fundamentais no processo de satisfação de necessidades. O recuento histórico em enfermagem, dá conta dos factos ou acontecimentos e personagens que em diferentes contextos marcaram pautas para o desenvolvimento da profissão.

$\mathrm{Na}$ actualidade, o estudo e ensino deste tópico, cobra especial importância como estratégia para avançar para o futuro com o reconhecimento dos antecedentes sociopolíticos e culturais que determinaram a sobrevivência, desenvolvimento e avanço da enfermagem como profissão e disciplina.

No presente texto, faz-se a descrição das actuações do Pai Pedro Claver durante seu estadía na Cartagena colonial, e analisa-se mediante um marco epistemológico a perspectiva filosófica do cuidado desenvolvida por ele durante sua missão de serviço aos negros escravos, e que ao longo do 
tempo influenciaram o desenvolvimento da enfermagem colombiana.

Descritores: Enfermagem, Pesquisa em Enfermagem, História da Enfermagem.

\section{RESUMEN}

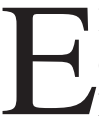
1 desarrollo histórico de la enfermería ha estado íntimamente ligado a la historia de la humanidad, por cuanto el cuidado de la vida y la salud, han sido actividades fundamentales en el proceso de satisfacción de necesidades. El recuento histórico en enfermería da cuenta de los hechos o acontecimientos y personajes que en diferentes contextos han marcado pautas para el desarrollo de la profesión.

En la actualidad, el estudio y enseñanza de la historia, cobra especial importancia como estrategia para avanzar hacia el futuro con el reconocimiento de los antecedentes sociopolíticos y culturales que han determinado la supervivencia, desarrollo y avance de la enfermería como profesión y disciplina.

En el presente texto, se hace la descripción de las actuaciones del Padre Pedro Claver durante su estadía en la Cartagena colonial y se analiza mediante un marco epistemológico la perspectiva filosófica del cuidado desarrollada por él durante su misión de servicio a los esclavos de raza negra que a lo largo del tiempo han influenciado el desarrollo de la enfermería colombiana.

Palabras clave: Enfermería, Investigación en Enfermería, Historia de la Enfermería.

\section{INTRODUCCIÓN}

Collière (1993), ha definido el cuidado como un acto humano que busca promover la vida y conservar la especie, en tanto que Castrillón (1997), se refiere a él como una práctica social que ha identificado y favorecido el desarrollo de la disciplina de enfermería. Es precisamente la respuesta social al encargo de cuidar, la que le ha permitido a esta disciplina reconstruir su historia como un elemento clave para entender el estado actual del cuidado profesional en las dimensiones científico- técnica, cultural y política y su proyección hacia el futuro en una sociedad globalizada y con una compleja problemática que incide sobre el desarrollo y por ende sobre la salud y la vida de las personas.

En este trabajo, se hace la revisión y análisis de algunas obras biográficas de San Pedro Claver con un marco epistemológico de enfermería determinado por las características de perspectiva disciplinar definidos por Afaf Meleis (1987), los patrones de conocimiento propuestos por Barbara Carper (1978) y las visiones del mundo de la enfermería formuladas por Newman (1991) y Fawcett (1995), con la pretensión contribuir al reconocimiento de la historia como el soporte fundamental para la identidad y proyección profesional hacia el futuro.

\section{OBJETIVOS}

Identificar los rasgos esenciales de perspectiva disciplinar, patrones de conocimiento y visiones del mundo de la enfermería que conforman el marco epistemológico en algunos relatos biográficos de San Pedro Claver, como uno de los personajes claves en el desarrollo histórico de la enfermería en Colombia.

\section{MATERIALES Y MÉTODOS}

Este trabajo se realizó mediante la revisión de fuentes secundarias sobre la vida de San Pedro Claver. Para ello se hizo lectura e interpretación de textos e identificación de conceptos que concordaran con las características de perspectiva disciplinar, los patrones de conocimiento y las visiones del mundo de la enfermería, que se estructuraron como categorías principales para el análisis de la información.

La búsqueda documental se llevó a cabo en la Biblioteca Nacional de España de la ciudad de Madrid, utilizando como herramienta el catálogo público institucional y empleando como palabra clave: San Pedro Claver; se obtuvieron 38 archivos entre libros de texto y retratos en las dos sedes de la Biblioteca ubicadas en Recoletos y Alcalá de Henares; posteriormente, se hizo una depuración de escritos tomando como criterios: que fuera un texto en Español con más de 30 años de haber sido publicado y disponible en el momento. Finalmente se hizo la revisión y lectura de 12 libros que correspondían a los criterios mencionados. 
Posteriormente, se llevó a cabo una lectura analítica y transcripción de algunos textos que permitieron identificar las características de la perspectiva disciplinar de enfermería. En cumplimiento de los asuntos éticos, se ha tratado de respetar la propiedad intelectual haciendo las respectivas referencias de los autores.

\section{RESULTADOS}

Los datos derivados de las lecturas fueron agrupados en las categorías: marco epistemológico de enfermería, historia de enfermería, biografía de San Pedro Claver y perspectiva disciplinar en las actuaciones del Santo. A continuación se hace referencia a cada una de ellas:

\section{MARCO ESPISTEMOLÓGICO ENFERMERÍA}

DE

El marco epistemológico de enfermería pretende mostrar la evolución del conocimiento y el significado de la disciplina en cuanto a la perspectiva, el dominio, los conceptos y los patrones de conocimiento (Durán, 2002). A su vez, la perspectiva disciplinar está definida por la naturaleza de la enfermería como una ciencia humana, los asuntos de la práctica, las relaciones establecidas entre los actores del cuidado y las perspectivas que los mismos tienen sobre la salud y el bienestar (Carper, 1978) (Durán, 2002).

Por otro lado, las visiones del mundo de la enfermería, como componentes del marco epistemológico, orientan la forma cómo deben comportarse los miembros de la disciplina en relación con la naturaleza humana de las personas y el propósito de la enfermería como ciencia humanística, comprendiendo los puntos de vista ontológico y epistemológico de la disciplina, y que han sido agrupadas por Fawcett (1995) y Newman (1991), en: las visiones particular-determinística o de reacción, integrativa-interactiva o de reciprocidad y visión humanitaria-transformativa o de simultaneidad (Durán, 2002).

Como soporte del desarrollo disciplinar, los patrones de conocimiento, han sido definidos como las formas propias de lograr y aprehender el conocimiento implícito en los fenómenos del cuidado de enfermería, que se han agrupado en el patrón empírico, referido al componente científico para la explicación y predicción de los fenómenos; el ético, que reúne los asuntos de la moral en la práctica; el patrón estético que evoca el componente artístico de la enfermería y el patrón de conocimiento personal que se relaciona con el ser de cada persona y que es la base fundamental para la puesta en marcha de todos los demás patrones enunciados (Carper, 1978) (Durán, 2002).

\section{HISTORIA DE ENFERMERÍA Y RELEVAN- CIA PARA EL DESARROLLO DISCIPLI- NAR}

Siles (1999) plantea que la historia de la enfermería se refiere a los hechos, sucesos, eventos o acontecimientos que tienen lugar en un tiempo dado y que constituyen el estudio historiográfico conformado por los escritos, estudios, análisis, resultados, explicaciones y conclusiones que constituyen la producción historiográfica en enfermería.

Desde esta perspectiva, el mismo autor (Siles, 1999), ha enfatizado en el carácter histórico de la práctica de los cuidados como componentes de la compleja realidad humana en el proceso de satisfacción de necesidades. El estudio de la historia de enfermería es un componente disciplinar relativamente joven, que ha significado un arduo trabajo de académicos e investigadores inquietos en el tema, con el propósito de aportar para la construcción de la identidad de la enfermería como práctica social en un contexto histórico y socio político dado. La consolidación del corpus de conocimientos y el reconocimiento de la historia como elemento disciplinar profesional son elementos fundamentales para los desarrollos teóricos a los problemas de la actualidad y contribuyan al mejoramiento de las prácticas de cuidado en el futuro.

De acuerdo con Velandia (1995), en el contexto colombiano, es escasa la producción historiográfica en enfermería, y los primeros ensayos históricos fueron hechos por médicos, situación que ha incidido desfavorablemente en la construcción de la historia en relación con el proceso histórico social del país; a su vez, esta situación ha hecho mella en el asunto de la identidad profesional y el desarrollo disciplinar. En consecuencia, la plataforma histórica se ha construido sobre los acontecimientos que han constituido la enfermería 


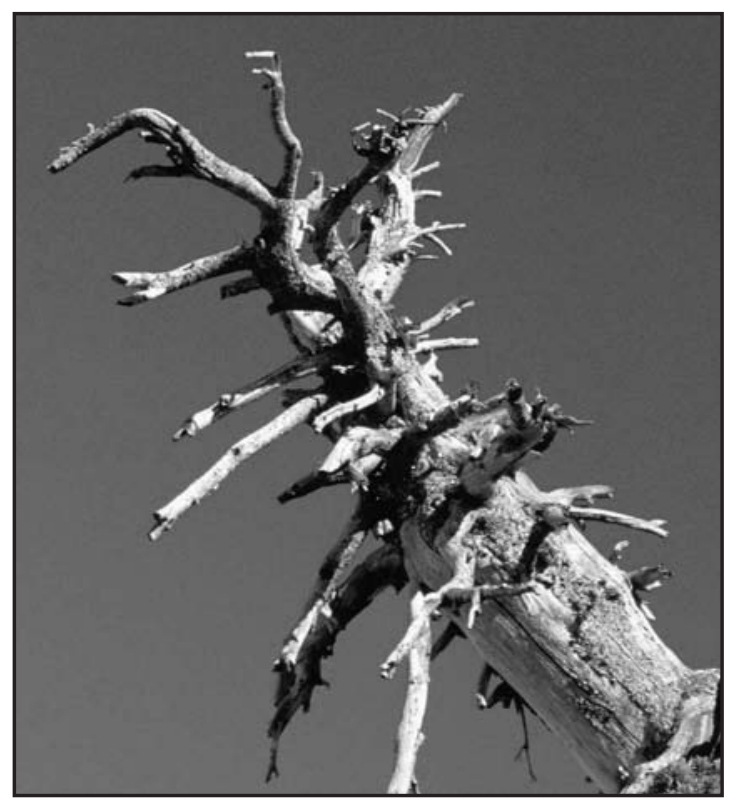

moderna a partir de la segunda mitad del siglo XIX, bajo la influencia de las órdenes religiosas de España, los trabajos de F. Nigthingale y los desarrollos investigativos y teóricos de las enfermeras norteamericanas.

$\mathrm{Al}$ analizar los antecedes históricos de la enfermería en Colombia, Velandia (1995), ha hecho referencia a cuatro elementos residuales o herencias recibidas por tradición: femenina, militar, religiosa y etnográfica. En cuanto a la herencia religiosa, ubica a San Pedro Claver como el "padre de la enfermería en Colombia" a raíz de su labor con los negros esclavos por haber ejercido una práctica de medicina y enfermería empíricas junto a su apostolado en el Hospital de San Lázaro al lado del Cerro de la Popa en Cartagena de Indias. Al lado de las actuaciones del Padre Claver, el trabajo desarrollado por órdenes religiosas como la de los Hermanos de San Juan de Dios, dieron inicio al desarrollo de la enfermería en el contexto nacional que a pesar de ser una disciplina social laica, conserva aún algunos rasgos de ésa herencia.

\section{BIOGRAFÍA DE SAN PEDRO CLAVER}

La Cartagena de Indias de la colonia, ciudad mercantil y punto clave para la estrategia militar fue el contexto en el que se desarrolló gran parte de la obra misional de San Pedro Claver en América, sin embargo, no ofreció el ambiente propicio para la conservación de archivos que documentaran tales hechos. De los archivos de la Compañía de Jesús existentes en España y América, se puede conocer lo que el Padre Astraín relata y que da cuenta de la lucha social que suscitó el apostolado del Santo, en medio de la adversidad impuesta por visitadores y examinadores del clero y la inquisición (Picón, 1969).

Con el objeto de entender los alcances de la obra de San Pedro Claver, es importante mirar algunos aspectos de su vida, el entorno en el que transcurrió y las influencias recibidas que lo motivaron a llevar a cabo su misión de apostolado en el cuidado de las personas más desvalidas que tenían la condición de ser inmigrantes, negros y esclavos en la Cartagena de Indias del siglo XV.

Por esta razón, resulta importante mencionar que San Pedro Claver vivió en una época brillante (1580 -1654), momento sociopolítico crucial en España, tiempo en el que sucedió la restauración católica; además el hecho de haber crecido en el seno de una familia de agricultores con marcado arraigo a la tradición religiosa católica, que se vio abocada a enfrentar dos acontecimientos dolorosos que influyeron para decidir su orientación religiosa y que le dejaron en su rostro una impresión de tristeza que lo acompañó durante toda su vida (Valtierra, 1954).

Nació en Verdú (España) el 26 de Junio de 1580 , en el seno de una familia integrada por sus padres, un hermano y dos hermanas. De su infancia se tienen pocos datos. Inició sus estudios bajo la tutoría inicial de su tío paterno Juan Claver y luego se dirigió a Barcelona en 1596. A los 16 años demostró su vocación por la vida religiosa, razón por la cual luego de sus estudios de retórica ingresó al Colegio de los Jesuitas para estudiar Filosofía en 1601; sobresalía entre sus compañeros por su ingenio y aplicación de igual manera, según testimonio del Obispo de Barcelona, Don Alonso Coloma "se hallaba más aventajado que los demás, no solo en Ciencias, sino también en piedad y religión (Valtierra, 1954).

Existen relatos de que pasaba largas horas en la Iglesia del Colegio y que cada domingo iba con algunos de sus compañeros al Hospital de Santa Cruz a cuidar los enfermos más miserables. Son estos los rasgos iniciales de su interés por prestar 
un servicio a las personas en sufrimiento y dolor, como lo hacen los enfermeros hoy en día para cuidar a sus pacientes, para lo que es necesario ingenio y creatividad, formación científica entrega a su labor (Valtierra, 1954).

Como rasgos fundamentales de su carácter y personalidad se destacaban su afabilidad, amabilidad, humildad y obediencia; era descrito como un ser calmado y retraído que tenía por norma general de vida la observancia fiel y total de las reglas (Valtierra, 1954).

Entró en la Compañía de Jesús en 1602, hizo sus votos como miembro el 8 de Agosto de 1604; a partir de esa fecha inició un largo recorrido en su ruta misionera, que incluyó Barcelona, Gerona, Mallorca, Tarragona y finalmente Cartagena de Indias, en el Nuevo Reino de Granada (Fernández, 1666) (Valtierra, 1954).

Se embarcó en 1610 desde el puerto de Sevilla en el galeón San Pedro rumbo al Nuevo Mundo. Terminó sus estudios en Bogotá, luego se dirigió a Tunja y finalmente fue llamado a Cartagena de Indias, a suplir al Padre Sandoval en el ministerio con los esclavos negros. En esta ciudad pudo conocer de cerca la realidad vivida en los barracones con cuerpos enfermos hacinados y que le sirvió de motivo para iniciar su labor de apostolado al servicio de estos esclavos (Fernández, 1666) (Valtierra, 1954).

Su ordenación ocurrió el 19 de Marzo de 1616, como el primer sacerdote jesuita investido en Cartagena de Indias. Murió el 8 de Septiembre de 1654, en la ciudad de Cartagena. Hacia 1747, sus virtudes fueron declaradas como heróicas, recibió la beatificación el 20 de Julio de 1850 y fue canonizado el 15 de Enero de 1888 (Fernández, 1666) (Valtierra, 1954).

Fueron diversas las acciones emprendidas por el Santo en favor de los esclavos y que han sido documentadas en las obras biográficas. Por su parte, comenta Valtierra (1954), que para cumplir su voto, asistía las necesidades espirituales y corporales de los negros sin miramientos de las distancias, las temperaturas o las dolencias; para tal efecto los instruía, los bautizaba, los confesaba y les asistía en el bien morir con tanto amor y caridad como si cada uno fuese su preferido. Les asistía en necesidades básicas como la nutrición, la comodidad y el vestido, todas ellas interferidas a causa de la larga y penosa travesía transatlántica.

Iba por todos los enfermos de uno en uno ofreciendo el Santo Cristo y preguntándoles si querían confesarse, para lo cual adoptaba posiciones que no fueran incómodas para ellos y no tanto para él. Como lo señala Valtierra (1954), “Todo el día el Padre Claver en un continuo movimiento, en un excesivo trabajo, en la congoja de un Hospital, entre vapores de enfermedades, sudando siempre de calor, y sobre todo eso ayunó todo el día" (p.54,57).

Amó el venerable Padre San Pedro Claver a los religiosos de la orden de San Juan de Dios por las obras que hacían con los enfermos y les manifestaba su interés en visitar sus instalaciones para aportar con su capacidad para cuidar a los enfermos, lo que significaba descanso para los religiosos. El Santo acudía varias veces por semana a los hospitales de San Lázaro, destinado para la atención a los leprosos, y al hospital de San Sebastián (denominado también San Juan de Dios), especialmente cuando estaban llenos para prestar su servicio en jornadas de trabajo que iban desde la mañana hasta la noche, bajo la subordinación del P. Prior o Enfermero hospitalario (Fernández, 1666) (Ortega, 1974).

De su servicio en el Hospital San Sebastián, se han documentado una serie de casos denominados "raros", que han servido para reconocer en el Padre su vocación y poder de sanación por medio de los milagros obrados en los pacientes con base en la fe y devoción. Un relato señalaba la participación de San Pedro Claver en el cuidado de unos marineros que habían ingresado luego de sufrir quemaduras graves, las cuales a causa de la exudación desprendían un fuerte olor, para lo que el Padre les brindaba comodidad ahuyentando las moscas y tábanos que les molestaban (Fernández, 1666 ) (Ortega, 1974 ).

Otro narración aludía a que en la sala de calenturas del mismo Hospital, el ciego Juan Ramírez adolecía de fuertes dolores de cabeza, ante lo cual el Santo brindaba consuelo dándole a entender que su ceguera podría ser un medio para salvar su alma y le cubría con su manta para aliviarle el dolor; además le daba un par de besos de paz en las mejillas, intervenciones que fueron efectivas para 
curarle la dolencia. Han sido muchas otras historias, entre ellas la que se menciona al señor Alvaro Barbosa Salazar, quien aislado por las lesiones luéticas dio cuenta de su sanación corporal y espiritual, al igual que otros pacientes en la sala de unciones mercuriales del Hospital San Sebastián por cuenta de la labor apostólica del Padre (Fernández, 1666) (Ortega, 1974).

En muchas ocasiones, antes de que las embarcaciones tocaran puerto, buscaba la manera de abordarlas y al llegar se ponía en medio de los negros manifestándoles su afecto. Por medio de los intérpretes les decía que venía para ser compañero de todos y les daba la bienvenida a la tierra de los blancos. Esta idea era necesaria, pues los esclavos creían que les traían para matarlos y de sus pieles hacer aceite para los navíos. Preguntaba por los enfermos y los niños, especialmente, por los que habían nacido durante el viaje transatlántico, esto con el fin de bautizarlos. Luego venía la repartición de regalos y el suministro de agua fresca; a los más decaídos, según relato de un testigo, les daba un remedio que les sacaba de su letargo, un buen vaso de agua fuerte o aguardiente (Valtierra, 1954).

A los enfermos atendía de modo delicado y asiduo. Como los desgraciados esclavos habían de quedar almacenados (como mercancías) en depósitos instalados a tal efecto (que no tenían muchas veces las condiciones requeridas de salubridad), el Padre Claver vigilaba a los más castigados para evitar infecciones y contagios (Fernández, 1666) (Valtierra, 1954).

A pesar de que su corazón temblaba ante la herejía, San Pedro Claver, tuvo un corazón lleno de benevolencia para los hombres herejes y les suministraba los cuidados que fueran necesarios, porque ante todo, primaba la dignidad humana como elemento fundamental de la doctrina católica (Valtierra, 1954).

\section{PERSPECTIVA DISCIPLINAR EN LAS ACTUACIONES DE SAN PEDRO CLAVER.}

Tomando como referencia los elementos de perspectiva disciplinar descritos antes: patrones de conocimiento (Carper, 1978), visión de la realidad (Newman, 1991) y perspectiva disciplinar (Fawcett, 1995), en las actuaciones de San Pedro Claver se distinguen los siguientes:

\section{PATRONES DE CONOCIMIENTO}

- Empírico: al utilizar de manera lógica sus conocimientos sobre higiene corporal, los principios básicos de terapéutica y de satisfacción de necesidades corporales (alimento, líquidos, mantenimiento de la temperatura, afecto) en la restitución de la salud.

- Estético: al tratar de representar, interpretar e imaginar la realidad y las situaciones vividas por los negros en su condición de esclavos, arrancados de su territorio, llevados en contra de su voluntad a otros lugares y mercantilizados como objetos para el trabajo. Esta forma de conocimiento, le permitió al santo acercarse de manera hermenéutica a la realidad de los esclavos.

- Ético: en razón de su condición y sustentado en los valores morales de la religión, el profundo sentido del respeto, la justicia y la beneficiencia.

- Personal: al darse cuenta y tratar de experimentar las situaciones vividas por los esclavos, el santo, ideó los mecanismos para aproximarse a la realidad y llevar a cabo las intervenciones que hoy le hacen merecedor de reconocimiento.

\section{VISIÓN DE LA REALIDAD DE ENFERMERÍA}

Las acciones llevadas a cabo por el santo y su forma de interacción con los esclavos, permiten identificar que San Pedro Claver tenía una visión integrativa-interactiva o de reciprocidad del mundo de la enfermería, en razón de que prestaba especial atención a la condición humana de los esclavos, interactuaba con ellos y reconocía algunos aspectos de las respuestas culturales en relación con los asuntos de la salud.

\section{PERSPECTIVA DISCIPLINAR}

Si bien San Pedro Claver dedicó su vida al ministerio religioso y su labor al apostolado con los negros esclavos, al analizar su biografía puede indicarse que su labor tenía algunos rasgos de la perspectiva disciplinar de enfermería, en virtud de que reconocía el carácter humano de su labor apostólica, llevaba a cabo acciones para promover la dignidad humana, la vida y restituir la salud y el bienestar de los esclavos y establecía con ellos unas relaciones más profundas que el acto terapéutico en sí. 


\section{DISCUSIÓN - CONCLUSIONES}

San Pedro Claver ha sido reconocido por su labor apostólica a favor de los negros esclavos llevados de Africa a América en el siglo XV; su labor evangelizadora se extendió más allá del campo de la religión y ante el sufrimiento y dolor que pudo evidenciar, llevó a cabo acciones de cuidado encaminadas a la restitución de la salud y la comodidad de los esclavos. Estas labores de cuidado, inspiraron de igual manera, el trabajo de diversas comunidades religiosas de origen español que por varios siglos ejercieron las labores de enfermería en todo el territorio colombiano, elevándolo a la categoría de "Padre de la Enfermería Colombiana".

La revisión de la obra de San Pedro Claver, bajo la perspectiva disciplinar de enfermería y tomando como referente el marco epistemológico actual, permite entender que la influencia del santo va más allá del carácter religioso que hasta ahora se le ha reconocido, por cuanto su labor de cuidado estaba estructurada en las formas o patrones de conocimiento empírico, personal, ético y estético y la visión de los esclavos como seres humanos con quienes se podía interactuar; además porque con su labor proyectaba el cuidado como una práctica social que requería soporte científico y con un elevado sentido humanístico.

Esta revisión y reflexiones iniciales, constituyen por tanto un punto de partida para la realización a futuro de indagaciones mejor estructuradas que permitan reconstruir la historia de la enfermería colombiana para fortalecer la identidad profesional y proyectar la enfermería como una práctica social enfocada al cuidado de la vida y a la promoción del desarrollo humano con justicia social.
Es un reto prioritario en el contexto colombiano la creación de líneas de investigación que promuevan la reconstrucción histórica, como estrategia para definir el rol de los profesionales de enfermería como agentes sociales de cambio.

\section{REFERENCIAS BIBLIOGRÁFICAS}

- Carper, B. (1978). Fundamental patterns of knowing in nursing. Advances in Nursing Science, 1(1): 13-24.

- Castrillón, M. (1997). La dimensión social de la práctica de la enfermería. Medellín: Editorial Universidad de Antioquia.

- Collière, M. (1993). Promover la vida. De la práctica de las mujeres cuidadoras a los cuidados de enfermería. Madrid: Interamericana McGraw-Hill.

- Durán, M. (1998). Enfermería: desarrollo teórico e investigativo. Bogotá: Unibiblos.

- Durán, M. (2002). Marco Epistemológico de la enfermería. Aquichán, 2(2): 7-18.

- Fawcett, J. (1987). Analysis and evaluation of conceptual models. Philadelphia: F A Davis Company.

- Fernández, J. (1666). Trabajos gloriosos y casos raros en el Hospital de San Sebastián. En: Fernández, J. Apostólica y penitente vida del venerable Padre Pedro Claver de la Compañía de Jesús. Zaragoza.

- Meleis, A. (1987). Theorethical Nursing Development and progress. Philadelphia: J. B. Lippincott.

- Newman, M., Sime, A., Corcoran-Perry, S. (1991) The focus of the discipline of nursing. Advances in Nursing Science, 14(1): 1-6.

- Ortega, L. (1974). Los Hermanos de San Juan de Dios en los Hospitales de Cartagena de Indias, años 1596 a 1835. Boletín Informativo. Madrid: Curia Provincial de Castilla.

- Picón, M. (1969). Pedro Claver: el Santo de los Esclavos. Madrid: Editorial Revista de Occidente.

- Siles, J. (1999). Historia de la Enfermería. Alicante: Editorial Aguaclara.

- Valtierra, A. (1954). San Pedro Claver S.J. El esclavo de los esclavos 1580-1654. Biografía ilustrada. Bogotá: Antares.

- Velandia, A. (1995). Historia de la enfermería en Colombia. Bogotá: Editorial Universidad Nacional de Colombia.

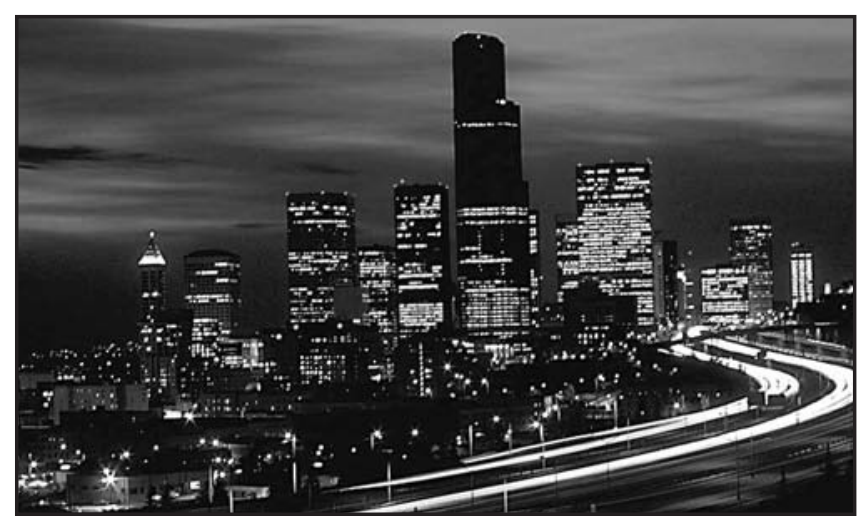

